\title{
Endothelium-Independent Vasodilatory Effect of Sailuotong (SLT) on Rat Isolated Tail Artery
}

\author{
S. Y. Yeon $\mathbb{D}^{1}{ }^{1}$ S. W. Seto $\mathbb{D}^{1,2}$ G. H. H. Chan, ${ }^{3}$ M. Low ${ }^{1},{ }^{1}$ H. Kiat $\mathbb{D}^{4,5,6}$ N. Wang, ${ }^{1,7}$ J. Liu, ${ }^{1,8}$ \\ and D. Chang $\mathbb{1}^{1}$ \\ ${ }^{1}$ NICM Health Research Institute, Western Sydney University, Penrith, NSW 2751, Australia \\ ${ }^{2}$ Department of Applied Biology and Chemical Technology, The Hong Kong Polytechnic University, Hung Hom, Kowloon, \\ Hong Kong SAR, China \\ ${ }^{3}$ Hong Kong Community College, The Hong Kong Polytechnic University, Hung Hom, Kowloon, Hong Kong \\ ${ }^{4}$ Faculty of Medicine, University of New South Wales, Kensington, NSW 2052, Australia \\ ${ }^{5}$ School of Medicine, Western Sydney University, Penrith, NSW 2571, Australia \\ ${ }^{6}$ Faculty of Medicine and Health Sciences, Macquarie University, Sydney, NSW 2113, Australia \\ ${ }^{7}$ Key Laboratory of Chinese Medicinal Formula of Anhui Province, Anhui University of Chinese Medicine, Hefei 230012, China \\ ${ }^{8}$ Xiyuan Hospital, China Academy of Chinese Medical Sciences, Beijing, China
}

Correspondence should be addressed to S. W. Seto; saiwang.seto@polyu.edu.hk

Received 15 June 2020; Revised 21 August 2020; Accepted 7 September 2020; Published 22 September 2020

Academic Editor: Silvia Wein

Copyright (c) 2020 S. Y. Yeon et al. This is an open access article distributed under the Creative Commons Attribution License, which permits unrestricted use, distribution, and reproduction in any medium, provided the original work is properly cited.

\begin{abstract}
Background. Sailuotong (SLT) is a standardized three-herb formulation consisting of extracts of Panax ginseng, Ginkgo biloba, and Crocus sativus for the treatment of vascular dementia (VaD). Although SLT has been shown to increase cerebral blood flow, the direct effects of SLT on vascular reactivity have not been explored. This study aims to examine the vasodilatory effects of SLT and the underlying mechanisms in rat isolated tail artery. Methods. Male (250-300 g) Wistar Kyoto (WKY) rat tail artery was isolated for isometric tension measurement. The effects of SLT on the influx of calcium through the cell membrane calcium channels were determined in $\mathrm{Ca}^{2+}$-free solution experiments. Results. SLT $(0.1-5,000 \mu \mathrm{g} / \mathrm{ml})$ caused a concentration-dependent relaxation in rat isolated tail artery precontracted by phenylephrine. In the contraction experiments, SLT $(500,1,000$, and $5,000 \mu \mathrm{g} / \mathrm{mL})$ significantly inhibited phenylephrine $(0.001$ to $10 \mu \mathrm{M})$ - and $\mathrm{KCl}(10-80 \mathrm{mM})$-induced contraction, in a concentration-dependent manner. In $\mathrm{Ca}^{2+}$-free solution, SLT $(500,1,000$, and $5,000 \mu \mathrm{g} / \mathrm{mL})$ markedly suppressed $\mathrm{Ca}^{2+}$-induced $(0.001-3 \mathrm{mM})$ vasoconstriction in a concentration-dependent manner in both phenylephrine $(10 \mu \mathrm{M})$ or $\mathrm{KCl}(80 \mathrm{mM})$ stimulated tail arteries. L-type calcium channel blocker nifedipine $(10 \mu \mathrm{M})$ inhibited PE-induced contraction. Furthermore, SLT significantly reduced phenylephrine-induced transient vasoconstriction in the rat isolated tail artery. Conclusion. SLT induces relaxation of rat isolated tail artery through endothelium-independent mechanisms. The SLT-induced vasodilatation appeared to be jointly meditated by blockages of extracellular $\mathrm{Ca}^{2+}$ influx via receptor-gated and voltage-gated $\mathrm{Ca}^{2+}$ channels and inhibition of the release of $\mathrm{Ca}^{2+}$ from the sarcoplasmic reticulum.
\end{abstract}

\section{Background}

Cerebrovascular diseases (CVDs), including ischemic stroke and vascular dementia $(\mathrm{VaD})$, are among the major causes of morbidity and mortality in developing and developed countries [1]. CVDs are strongly associated with a number of risk factors, such as hypertension, obesity, aging, diabetes, and hypercholesterolemia [2]. Altered vascular reactivity, such as impaired vasodilatation or enhanced vasoconstriction, has been considered one of the common characteristics associated with these risk factors [3-5], directly contributing to the development and progression of CVD. For example, impaired vascular relaxation has been shown to play a role in the development of $\mathrm{VaD}$ in deoxycorticosterone acetate (DOCA) salt-induced hypertensive rats [6]. Similarly, impaired carotid artery relaxation has been suggested as a 
contributor to stroke and dementia in aged mice [7]. Therefore, interventions that can induce vasodilation or suppress vasoconstriction could be useful in reducing the progression of CVD.

Sailuotong (SLT) is a standardized three-herb formula Chinese herbal medicine designed for the management of $\mathrm{VaD}$ associated with ischemic stroke $[8,9]$. The SLT formula consists of standard extracts of specific dosages of Panax ginseng C. A. Meyer (ginseng), Gingko biloba L. (ginkgo), and Crocus sativus L. (saffron). The chemical profile and ratio of the three herbal extracts had been determined and studied in detail previously $[10,11]$. The cognitive enhancing effect of SLT has been demonstrated in both preclinical and clinical studies. In a chronic cerebral hypoperfusion model induced by bilateral common carotid artery ligation in rats, eight-week treatment of SLT significantly suppressed hypoperfusion-induced cognitive impairments, and this change is associated with reduction of activity of cholinesterase and increased acetylcholine (ACh) levels and superoxide dismutase (SOD) activity the brain tissue [8]. Our previous work also showed that SLT protected cultured human vascular endothelial cells against hydrogen peroxideinduce injury via increase in SOD activity [12]. The potential beneficial effects of SLT for VaD were demonstrated in two separate phase II randomized, double-blinded, placebocontrolled clinical studies. In these studies, treatment with SLT was shown to significantly improve cognitive function and cerebral blood flow to the inferior frontal and anterior temporal lobes in patients with $\mathrm{VaD}[11,13]$.

Reports from animal and clinical studies have indicated that the clinically beneficial effect of SLT is, at least, partially associated with an increase in cerebral blood flow [14, 15]. However, the underlying mechanism(s) associated with these SLT-mediated cerebral blood flow increase remain to be determined. In this study, we hypothesised that SLT has direct modulatory effects in vascular reactivity. Therefore, this study was aimed to evaluate the vascular effects and the underlying mechanisms of SLT using rat isolated tail artery.

\section{Methods}

2.1. Chemical and Drugs. SLT extracts were provided in-kind by the Shineway Pharmaceutical Group (Shijiazhuang, China). Acetylcholine (ACh), phenylephrine, calcium chloride $\left(\mathrm{CaCl}_{2}\right)$, tetraethylammonium (TEA), clotrimazole, glibenclamide, potassium chloride $(\mathrm{KCl})$, ethylene glycolbis( $\beta$-aminoethyl ether)- $N, N, N^{\prime}, N^{\prime}$-tetraacetic acid (EGTA), $l$ - $N$-nitro arginine methyl ester (L-NAME), and nifedipine were purchased from Sigma-Aldrich (St Louis, MO, USA). All the other reagents were of analytical grade.

2.2. High-Performance Thin-Layer Chromatography (HPLC) Analysis of the SLT Formula. HPLC-PDA was employed to profile the phytochemical composition of the SLT extract used in the study. The HPLC-PDA analysis was performed on a Prominence-I LC-2030 3D Plus Shimadzu HPLC system controlled by Lab Solutions software (Shimadzu, Australia). Separation was achieved using a Shimadzu Shim- pack GIST (Shimadzu, Australia) reverse phase C18 column $(4.6 \times 150 \mathrm{~mm}$ I.D., $5 \mu \mathrm{m})$ maintained at $40^{\circ} \mathrm{C}$.

The SLT extract was dissolved by sonication in $30 \%$ aqueous acetonitrile for $30 \mathrm{~min}$ at $5 \mathrm{mg} / \mathrm{ml}$. Individual solutions of standards, crocin, ginsenoside Re, ginsenoside $\mathrm{Rg} 1$, ginsenoside $\mathrm{Rd}$, quercetin, kaempferol, and isorhamnetin were prepared to $1 \mathrm{mg} / 10 \mathrm{ml}$ in $30 \%$ aqueous acetonitrile for identification and combined for analysis. The sample and mixed standard were syringe-filtered with $0.45 \mu \mathrm{m}$ PTFE.

The SLT HPLC-PDA profiles were generated by $20 \mu \mathrm{l}$ injection. The mobile phase consisted of $0.1 \%(\mathrm{v} / \mathrm{v})$ aqueous formic acid (mobile phase A) and $0.1 \%(\mathrm{v} / \mathrm{v})$ formic acid in acetonitrile (mobile phase $\mathrm{B}$ ). The gradient program was $10 \%$ B for 1 min with a linear increase, to $45 \%$ B at $45 \mathrm{~min}$, and then, a wash and re-equilibration. The mobile phase flow rate was maintained at $1.1 \mathrm{ml} / \mathrm{min}$. The PDA was set to acquire absorbance data from 190 to $800 \mathrm{~nm}$.

2.3. Animals. A total of forty 12 -week-old, male Wistar Kyoto (WKY) rats weighting 200-250 g were obtained from the Animal Resource Centre (Canning Vale, Western Australia). All experimental animals were housed under a 12:12 hour light-dark cycle (relative humidity: 50-60\%; temperature: $22 \pm 1^{\circ} \mathrm{C}$ ) and were given standard chow and water ad libitum throughout the experimental period. On the day of experiment, the rats were sacrificed by $\mathrm{CO}_{2}$ asphyxiation, and vascular preparations were isolated under a dissection microscope. All animal protocols conformed to the Guide for the Care and Use of Laboratory Animals by the Australian Code of Practice for the Care and Use of Animals for Scientific Purpose [16]. Institutional ethics approval (Approval number: A12041) was obtained from Western Sydney University prior to commencement of the study.

2.4. Isometric Tension Measurement. Isometric force measurements and experimental protocols were performed on tail artery isolated from the WKY rats as described previously with slight modifications $[5,17]$. The isometric force of the arterial ring segments was measured using a multiwire myograph system (Model 620M; Danish Myo Technology, DMT, Denmark). During the isometric tension measurement, the chambers of the myograph were filled with Krebs-Henseleit solution (mmol/L: $\mathrm{NaCl} 118.0, \mathrm{KCl} 4.7$, $\mathrm{K}_{3} \mathrm{PO}_{4} 1.2, \mathrm{MgSO}_{4}$ 1.2, $\mathrm{NaHCO}_{3} 25.0, \mathrm{CaCl}_{2}$ 1.8, glucose 11.0), unless stated otherwise. The Krebs-Henseleit solution were prepared and used as follows: All solutions were warmed to $37^{\circ} \mathrm{C}$ and bubbled with carbogen $\left(95 \% \mathrm{O}_{2}\right.$ and $5 \%$ $\mathrm{CO}_{2}$ ) before they were added to the myograph chamber. Each chamber containing $5 \mathrm{ml}$ of Krebs buffered solution was bubbled with $95 \% \mathrm{O}_{2}$ and $5 \% \mathrm{CO}_{2}$ and maintained at a $\mathrm{pH}$ of 7.4 at $37^{\circ} \mathrm{C}$ throughout the experiment. Arterial rings (1.5-2.0 mm length) were mounted in the organ chambers, and normalized passive resting force and the corresponding diameter were determined for each preparation from its own length-pressure curve and stretched to their optimal lumen diameter (i.e., $\sim 90 \%$ of the diameter which is equivalent to a transmural pressure of $100 \mathrm{~mm} \mathrm{Hg}$ ). The rings were, then, 
left for a stabilization period of about 30 minutes until a stable base line tone was obtained before beginning of the experiment. The presence of the endothelium was tested with acetylcholine $(1 \mu \mathrm{M})$ after preconstriction with phenylephrine $(\mathrm{PE})(1 \mu \mathrm{M})$. Tissues which showed a relaxation to acetylcholine of less than $60 \%$ of the phenylephrine contraction were not considered with intact endothelium and, therefore, not employed in this study. Responses were recorded using computerized data acquisition and recording software.

\subsection{Experimental Protocols of Vascular Tension Studies}

2.5.1. Protocol 1: Vasodilatory Effect of the SLT Extract in Rat Isolated Tail Arteries. The acute and direct vasodilatory effect of SLT was evaluated on rat isolated tail artery. PE $(1 \mu \mathrm{M})$ was applied to induce a steady contraction. When the contraction reached a plateau, the SLT extract $(0.1-5000 \mu \mathrm{g} /$ $\mathrm{ml}$ ) was added cumulatively to the isolated tail artery rings with or without the presence of L-NAME $(20 \mu \mathrm{M})$ to obtain a concentration-response curve. The relaxation effect was calculated as the percentage of the contraction in response to PE.

2.5.2. Protocol 2: Effects of Potassium Channel Blockers on SLT-Induced Vasodilation in Rat Isolated Tail Arteries. Rat isolated tail arteries were preincubated with various $\mathrm{K}^{+}$ channel blockers: (1) nonselective $\mathrm{K}^{+}$channel blocker, tetraethylammonium (TEA, $1 \mathrm{mM}$ ); (2) selective ATP-sensitive $\mathrm{K}^{+}$channel $\left(\mathrm{K}_{\mathrm{ATP}}\right)$ blocker, glibenclamide $(3 \mu \mathrm{M})$, and (3) $\mathrm{Ca}^{2+}$-dependent $\mathrm{K}^{+}$channel $\left(\mathrm{K}_{\mathrm{Ca}}\right)$ blockers, clotrimazole $(5 \mu \mathrm{M})$. Each blocker was allowed to incubate with the preparation for $30 \mathrm{~min}$ before the construction of a concentration-response curve.

2.5.3. Protocol 3: Calcium-Induced Vasoconstriction in Rat Isolated Tail Arteries. The effect of SLT on calcium influx induced by two different agents was evaluated. The preparations were initially contracted with either $\mathrm{KCl}(80 \mathrm{mM})$ or $\mathrm{PE}(10 \mu \mathrm{M})$ to determine maximum contraction of each preparation in normal Krebs solution. The preparations were washed with a calcium-free solution (identical concentration of EGTA substituted for $\mathrm{Ca}^{2+}$ in a normal Krebs solution) until fully relaxed. To exhaust the intracellular calcium storage, the preparations were stimulated with either $\mathrm{PE}(10 \mu \mathrm{M})$ or $\mathrm{KCl}(80 \mathrm{mM})$ repeatedly, with calciumfree solution wash in between, until any contractile response disappeared. The preparations were, then, incubated with SLT at various concentrations $(500,1,000$, and $5,000 \mu \mathrm{g} / \mathrm{ml})$ for $30 \mathrm{~min}$. After incubation, a single dose of the contractile agent (either $\mathrm{KCl}(80 \mathrm{mM})$ or $\mathrm{PE}(10 \mu \mathrm{M})$ was added to the chamber, and calcium (0.001-3 mM)-induced contraction concentration response curves were constructed.

2.5.4. Protocol 4: Phenylephrine- and Potassium Chloride(KCl-) Induced Vasoconstriction in Rat Isolated Tail Arteries.
In order to assess the effects of SLT on the contraction induced by phenylephrine and potassium chloride $(\mathrm{KCl})$, concentration-response curves of $\mathrm{PE}$ and $\mathrm{KCl}$ were constructed with or without the presence of SLT at various dosages $(500,1,000$, and $5,000 \mu \mathrm{g} / \mathrm{ml})$. The arterial preparations were allowed to preincubate with $\operatorname{SLT}(0,500,1,000$, and $5,000 \mu \mathrm{g} / \mathrm{ml}$ ) for 30 minutes' prior the construction of the $\mathrm{PE}$ or $\mathrm{KCl}$-induced vasoconstriction concentration-response curve.

2.5.5. Protocol 5: Role of Intracellular Calcium-Induced Vasoconstriction in Rat Isolated Tail Arteries. To clarify the role of intracellular $\mathrm{Ca}^{2+}$ release from the sarcoplasmic reticulum (SR) in the relaxant effect of SLT, the isolated tail artery rings were exposed to a $\mathrm{Ca}^{2+}$-free solution for 15 minutes before the application of $1 \mu \mathrm{M}$ of $\mathrm{PE}$ to induce the first transient contraction (Con 1). The rings were, then, washed with a normal Krebs solution for three times and incubated in the normal Krebs solution for 45 minutes to allow refill of the intracellular $\mathrm{Ca}^{2+}$ storage. After the 45-minute incubation, the medium was rapidly replaced with a $\mathrm{Ca}^{2+}$-free solution and allowed to incubate for 15 minutes. The second contraction (Con 2) was induced by $1 \mu \mathrm{M}$ of PE with or with the presence of SLT $(500,1,000$, or $5,000 \mu \mathrm{g} / \mathrm{ml})$. The ratio of Con 2 to Con 1 was calculated.

2.6. Statistical Analysis. Data are expressed as mean$\mathrm{s} \pm$ S.E.M., and $n$ denotes the number of replications for each data point. Relaxation was expressed as the percentage of the contraction elicited by phenylephrine or $\mathrm{KCl}$. Statistical comparisons were performed using the $t$-test or two-way analysis of variance (ANOVA), where appropriate. Differences were considered to be statistically significant at $P<0.05$. All statistical analyses were performed using GraphPad Prism 5 software (GraphPad Software, Inc., USA).

\section{Results}

3.1. High-Performance Thin-Layer Chromatography (HPLC) Analysis of the SLT Extract. The active ingredients and contents in SLT have been reported in details previously $[10,11]$. Six major bioactive compounds were analyzed using HPLC. Similar to these studies, our results showed that all these major bioactive compounds, including ginsenosides $\mathrm{Rg} 1$, ginsenosides Re, ginsenosides Rb1, quercetin, isorhamnetin, and crocin, are present in the SLT extract (Figure 1). The chromatogram is visualized at different wavelengths corresponding to the optimal wavelength to detect the relevant group of markers. SLT is reported to contain ginsengs as ginsenosides Rg1 (2.4 mg/capsule), ginsenosides $\mathrm{Re}(1.6 \mathrm{mg} /$ capsule), and ginsenosides $\mathrm{Rb} 1$ ( $4.49 \mathrm{mg} /$ capsule). The ginsenosides were confirmed to be present in similar concentrations by matching retention time and the UV spectrum to the reference standards as shown in Figure 1 at $203 \mathrm{~nm}$. The peak size is not reflective of abundance as the different ginsenosides have unique molar absorptivity. SLT is reported to contain Ginkgo biloba, with 


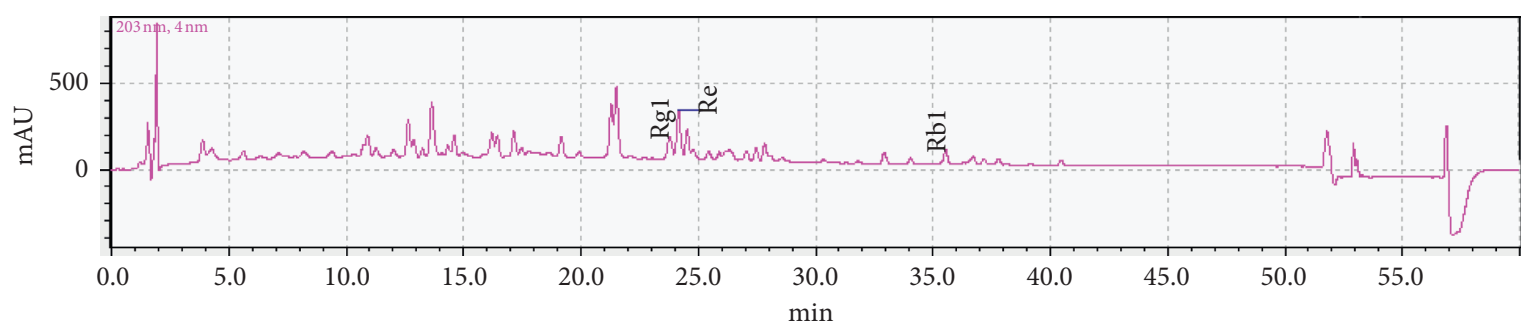

(a)

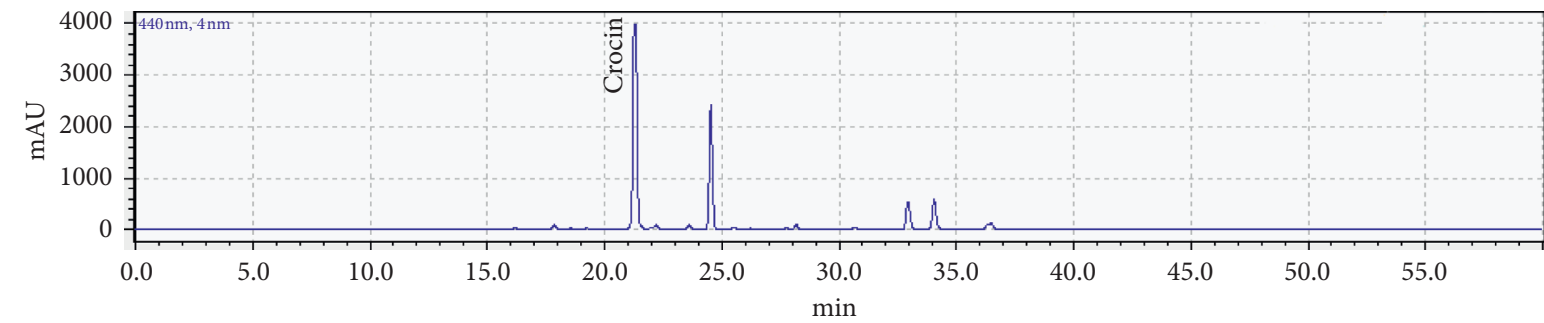

(b)

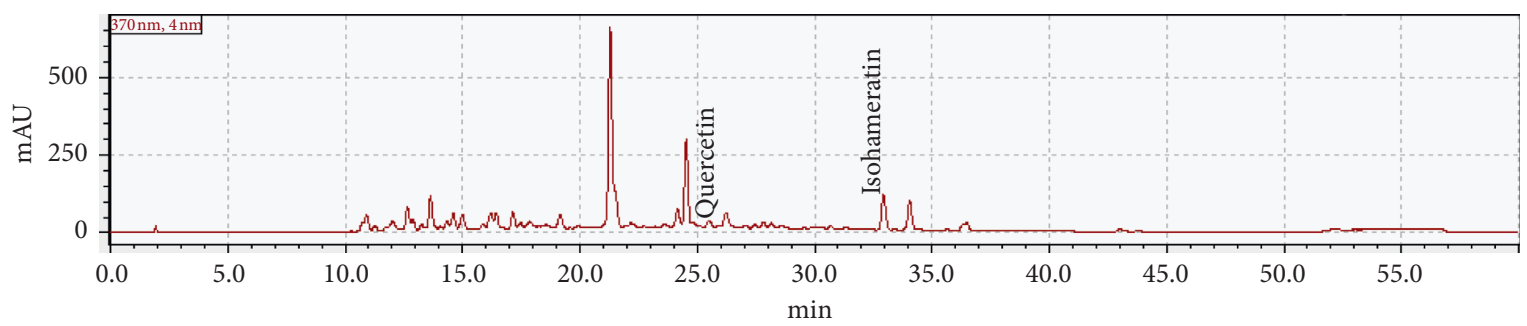

(c)

Figure 1: HPLC chromatograms of the SLT extract. The chromatogram (a) at $203 \mathrm{~nm}$ (pink) shows the ginsenosides Rg1, ginsenosides Re, and ginsenosides. The chromatogram (b) at $440 \mathrm{~nm}$ (blue) shows crocin. The chromatogram (c) at $370 \mathrm{~nm}$ (maroon) shows quercetin and isorhamnetin.

markers quercetin $(2.71 \mathrm{mg} /$ capsule) and isorhamnetin $(1.55 \mathrm{mg} /$ capsule). These were identified in the SLT extract used in this study as shown in Figure 1 at $370 \mathrm{~nm}$. SLT is reported to contain saffron, with the marker crocin $(1.7 \mathrm{mg} /$ capsule). Crocin was identified in the SLT extract used in this study as shown in Figure 1 at $440 \mathrm{~nm}$. The HPLC analysis of the SLT extract supplied by Shineway was consistent with other reports $[10,11]$.

\subsection{Vasodilatory Effects of the SLT Extract in Rat Isolated Tail} Arteries. SLT $(0.1-5,000 \mu \mathrm{g} / \mathrm{ml})$ caused a relaxation in the PE $(1 \mu \mathrm{M})$-preconstricted rat isolated tail artery in a concentration-dependent manner. The relaxation was initiated at $100 \mu \mathrm{g} / \mathrm{ml}$ and achieved $80.104 \pm 9.80 \%$ at $5,000 \mu \mathrm{g} / \mathrm{ml}$ $(n=6)$ (Figure 2). Preincubation of the isolated tail artery with L-NAME $(20 \mu \mathrm{M})$ did not significantly alter the SLTinduced relaxation $(n=6)$ (Figure 2).

\subsection{Effects of Potassium Channel Blockers on SLT-Induced} Vasodilatation in Rat Isolated Tail Arteries. Opening of potassium channels plays an important role in vasodilatation. We examined the involvement of potassium channels on the SLT-induced relaxation in the rat isolated tail arteries. Tetraethylammonium (TEA, a nonselective potassium

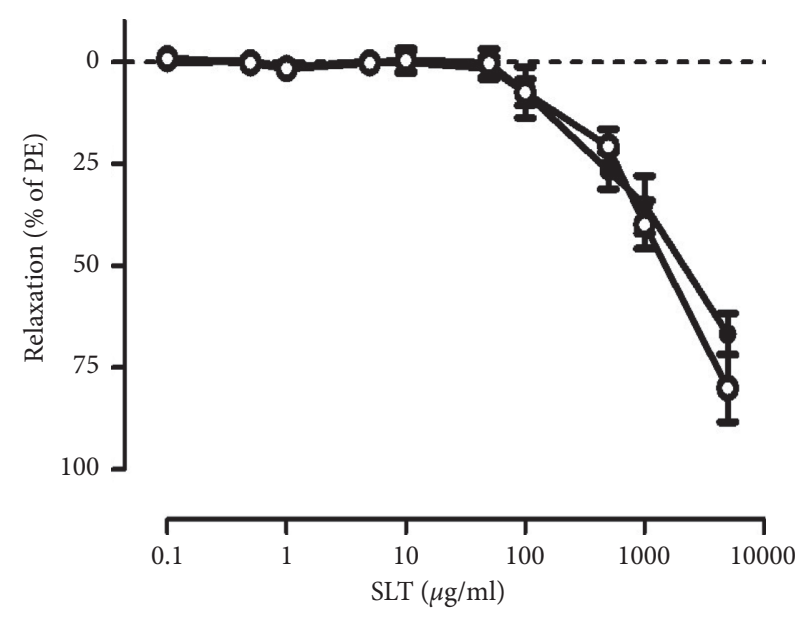

FIgURE 2: Cumulative concentration-response of the SLT extract $(0.1-5,000 \mu \mathrm{g} / \mathrm{ml})$ on phenylephrine $(1 \mu \mathrm{M})$-preconstricted rat isolated tail artery with $(\bullet)$ or without $(O)$ the presence of L-NAME $(20 \mu \mathrm{M})$. Values are expressed as mean \pm SEM $(n=6)$.

channel blocker) (1 mM), glibenclamide (Glib, an ATPsensitive potassium channel blocker) $(3 \mu \mathrm{M})$, and clotrimazole $\left(\mathrm{K}_{\mathrm{Ca}}\right.$, a calcium-activated potassium channel blocker) $(5 \mu \mathrm{M})$ were tested. As shown in Figure 2, preincubation of potassium channel blockers did not 
significantly alter the SLT-induced vasodilatation in PE $(1 \mu \mathrm{M})$-preconstricted rat isolated tail artery $(n=5-6)$ (Figure 3).

3.4. Effects of the SLT Extract on Calcium-Induced Vasoconstriction in Rat Isolated Tail Arteries Stimulated by Phenylephrine or KCl. The effect of the SLT extract on calcium-influx-induced vasoconstriction on rat isolated tail arteries was assessed with two vasocontractile stimulants (phenylephrine and $\mathrm{KCl}$ ) by reintroducing calcium (0.001-3 mM) into the calcium-free buffer. In the phenylephrine $(10 \mu \mathrm{M})$-stimulated tail arteries, cumulative administration of calcium chloride $(0.001-3 \mathrm{mM})$ induced a vasoconstriction in a concentration-dependent manner, with $29.167 \pm 7.23 \%$ contraction observed at $3 \mathrm{mM}(n=5)$. Preincubation of the SLT extract $(500,1,000$, and 5,000 $\mu \mathrm{g} /$ $\mathrm{ml}$ ) dose-dependently suppressed the calcium-induced vasoconstriction in the phenylephrine $(10 \mu \mathrm{M})$-isolated tail arteries. SLT at $5,000 \mu \mathrm{g} / \mathrm{ml}$ and nifedipine $(10 \mu \mathrm{M})$ markedly suppressed the calcium-induced vasoconstriction (Figure 4(a)).

Similarly, in the $\mathrm{KCl}(80 \mathrm{mM})$-stimulated tail arteries, cumulative administration of calcium chloride (0.001-3 mM) induced a vasoconstriction in the tail artery in a concentration-dependent manner, with $75.460 \pm 14.52 \%$ contraction observed at $3 \mathrm{mM}(n=6)$. Preincubation of the SLT extract at 1,000 and $5,000 \mu \mathrm{g} / \mathrm{ml}$, but not $500 \mu \mathrm{g} / \mathrm{ml}$, markedly inhibited the calcium-induced vasoconstriction in the $\mathrm{KCl}(80 \mathrm{mM})$-stimulated isolated tail arteries. SLT at $5,000 \mu \mathrm{g} / \mathrm{ml}$ abolished the calcium-induced vasoconstriction $(n=6)(P<0.001)$ (Figure 4(b)).

3.5. Effects of the SLT Extract on Phenylephrine- and KClInduced Vasoconstriction in Rat Isolated Tail Arteries. The effects of the SLT extract $(500,1,000$, and $5,000 \mu \mathrm{g} / \mathrm{ml})$ on phenylephrine-induced vasoconstriction in rat isolated tail arteries were evaluated. Cumulative addition of phenylephrine $(0.0001-10 \mu \mathrm{M})$ caused a vasoconstriction in the tail arteries in a concentration-dependent manner. Preincubation of the SLT extract at 1,000 and $5,000 \mu \mathrm{g} / \mathrm{ml}$, but not $500 \mu \mathrm{g} / \mathrm{ml}$, significantly suppressed the phenylephrineinduced vasoconstriction in the isolated tail arteries $(n=5)$ $(P<0.001)$. Nifedipine $(10 \mu \mathrm{M})$ markedly reduced the PEinduced vasoconstriction (Figure 5(a)). The vasoconstriction caused by phenylephrine at $10 \mu \mathrm{M}$ was markedly suppressed by $67.58 \%$ with the presence of the SLT extract at $5,000 \mu \mathrm{g} / \mathrm{ml}$ (control: $16.44 \pm 2.46 \mathrm{mN}$ vs. SLT: $5.33 \pm 1.02 \mathrm{mN})(P<0.05)$ $(n=5)$ (Figure 5(b)).

The effects of the SLT extract $(500,1,000$, and 5,000 $\mu \mathrm{g} /$ $\mathrm{ml}$ ) on $\mathrm{KCl}$-induced vasoconstriction in rat isolated tail arteries were evaluated. Cumulative addition of $\mathrm{KCl}$ $(10-80 \mathrm{mM})$ caused a vasoconstriction in the tail arteries in a concentration-dependent manner. Preincubation of the SLT extract at $500,1,000$, and $5,000 \mu \mathrm{g} / \mathrm{ml}$ significantly suppressed the $\mathrm{KCl}$-induced vasoconstriction in the isolated tail arteries in a dose-dependent manner. $(n=3)(P<0.001)$ (Figure 5(c)). The vasoconstriction caused by $80 \mathrm{mM}$ was abolished with the presence of the SLT extract at $5,000 \mu \mathrm{g} / \mathrm{ml}$

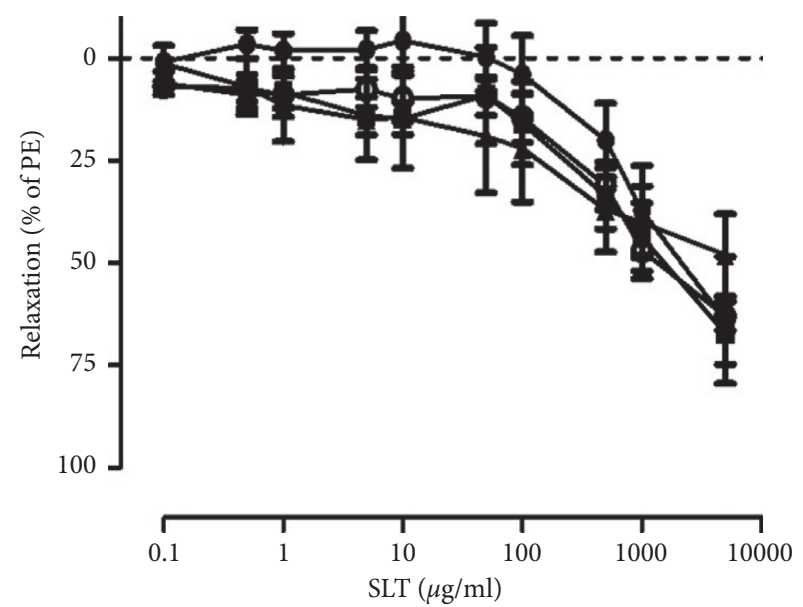

FIgURE 3: Effects of potassium channel blockers on SLT extractinduced relaxation on phenylephrine $(1 \mu \mathrm{M})$-preconstricted rat isolated tail artery. Tail arteries were preincubated with either vehicle control (O), tetrethyl-ammonium (TEA, a nonselective potassium channel blocker) $(1 \mathrm{mM})(\bullet)$, glibenclaminde (Glib, an ATP-sensitive potassium channel blocker) $(3 \mu \mathrm{M})(\mathbf{\square})$, or clotrimazole ( $\mathrm{KCa}$, a calcium-activated potassium channel blocker) $(5 \mu \mathrm{M})(\mathbf{\Delta})$ for 30 minutes before construction of the concentration-response curve. Values are expressed as mean \pm SEM $(n=5-6)$.

(control: $7.90 \pm 1.75 \mathrm{mN}$ vs. SLT: $0.54 \pm 0.31 \mathrm{mN})(P<0.05)$ $(n=3)$ (Figure 5(d)).

3.6. Effect of SLT on Intracellular Calcium Release. Phenylephrine (PE) $(1 \mu \mathrm{M})$ caused a transient contraction in the $\mathrm{Ca}^{2+}$-free solution through a release of intracellular $\mathrm{Ca}^{2+}$ from the sarcoplasmic reticulum. A second transient contraction was induced with or without the presence of SLT $(500,1,000$, and $5,000 \mu \mathrm{g} / \mathrm{ml})$. SLT significantly suppressed the PE-induced transient contraction ratio (Con 2/Con 1) at $5,000 \mu \mathrm{g} / \mathrm{ml} \quad(\mathrm{CLT}: \quad 39.12 \pm 6.28$ vs. SLT $5,000 \mu \mathrm{g} / \mathrm{ml}$ : $6.86 \pm 1.52)(P<0.05)(n=6)$ (Figure 6).

\section{Discussion}

Vascular dementia $(\mathrm{VaD})$ is considered to be caused by chronic cerebral ischemia which induces neuronal damage, resulting in a decline in cognitive function eventually. While the development and progression of cognitive impairment and $\mathrm{VaD}$ are certainly multifactorial, it has been suggested that cerebrovascular dysfunction leads to reduction of cerebral blood flow which is closely associated with cognitive impairment in diabetes [18, 19]. Sailuotong (SLT) is a standardized three-herb formula designed for the management of $\mathrm{VaD}$. Although data from animal and clinical studies have indicated that the clinically beneficial effect of SLT is closely associated with an increase in cerebral blood flow [11-13], the acute and direct modulatory effects and the underlying mechanisms of actions of SLT in vascular reactivity have not been studied. Results from the current work provide evidence that the SLT extract induces vascular relaxation in rat isolated tail arteries. Furthermore, our results showed that this SLT-induced vasodilatation is mediated via 


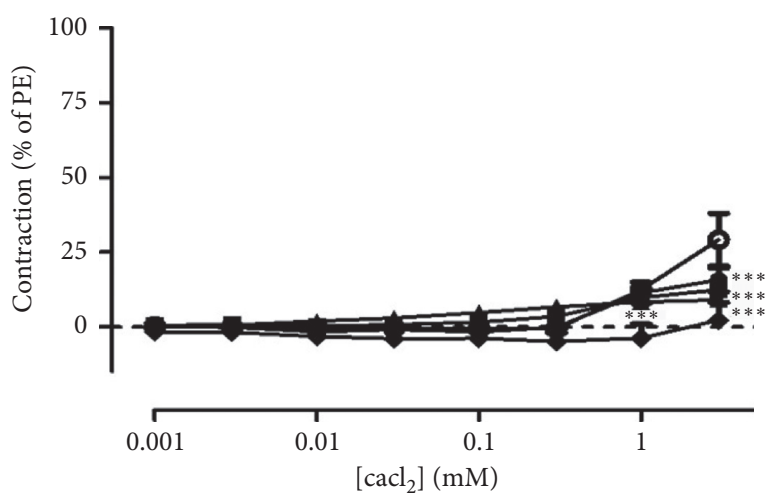

(a)

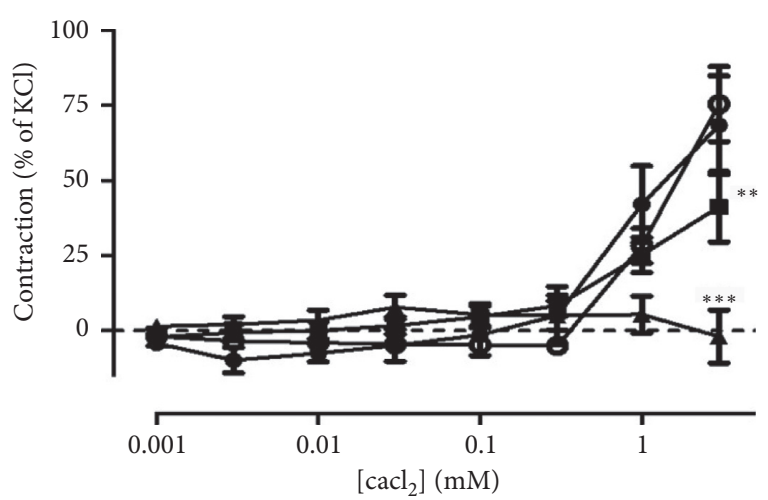

(b)

Figure 4: (a) Effect of the vehicle control (O), SLT extract $(500(\bullet), 1000(\boldsymbol{\square})$, or $5000(\mathbf{\Delta}) \mu \mathrm{g} / \mathrm{ml})$, or nifedipine $(10 \mu \mathrm{M})(\bullet)$ on Ca ${ }^{2+}$-induced vasoconstriction in phenylephrine $(\mathrm{PE})(10 \mu \mathrm{M})$-stimulated rat isolated tail arteries. Values are expressed as mean $\pm \mathrm{SEM}\left({ }^{* * *} P<0.001 \mathrm{vs}\right.$ Control) $(n=5)$. (b) Effect of the vehicle control $(O)$ and SLT extract $\left(500(\bullet), 1,000(\boldsymbol{\square})\right.$, or 5,000 $\left.(\mathbf{\Delta}) \mu \mathrm{g} / \mathrm{ml}^{\prime}\right)$ on Ca ${ }^{2+}$-induced vasoconstriction in potassium chloride $(\mathrm{KCl})(80 \mathrm{mM})$-stimulated rat isolated tail arteries. Values are expressed as mean $\pm \mathrm{SEM}\left({ }^{* *} P<0.01 \mathrm{vs.}\right.$ control ${ }^{* * *} P<0.001$ vs. control) $(n=5-8)$.

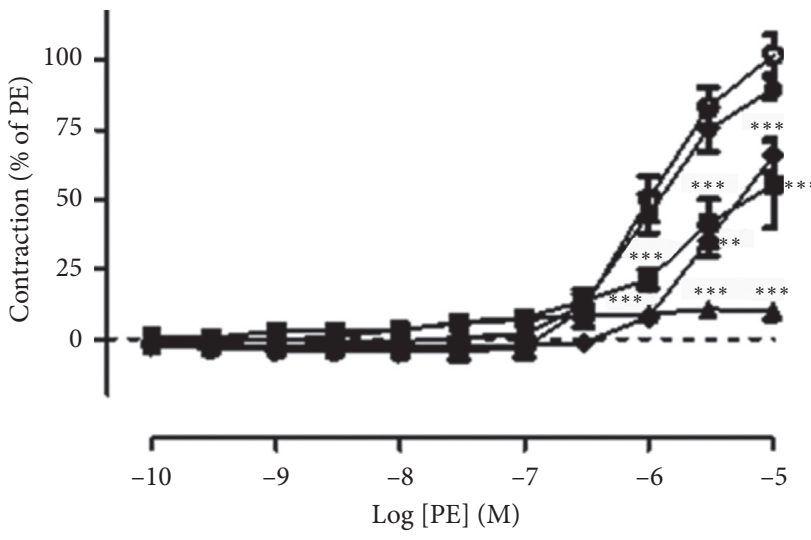

(a)

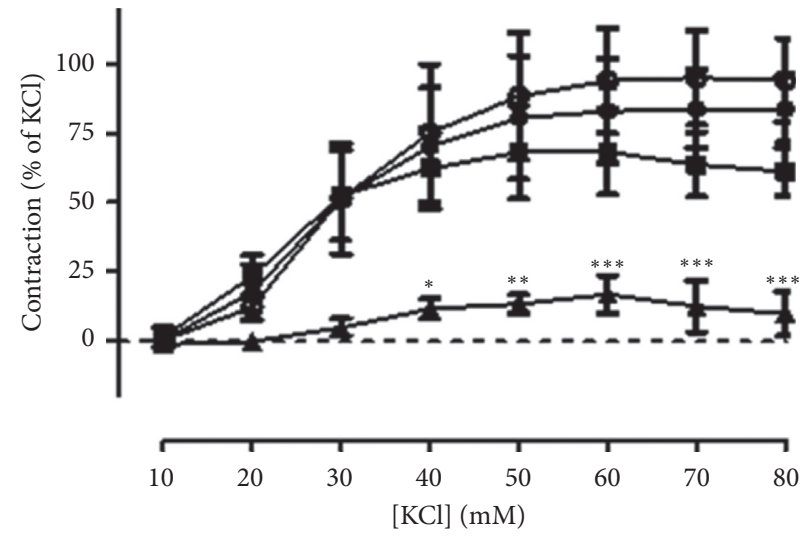

(c)

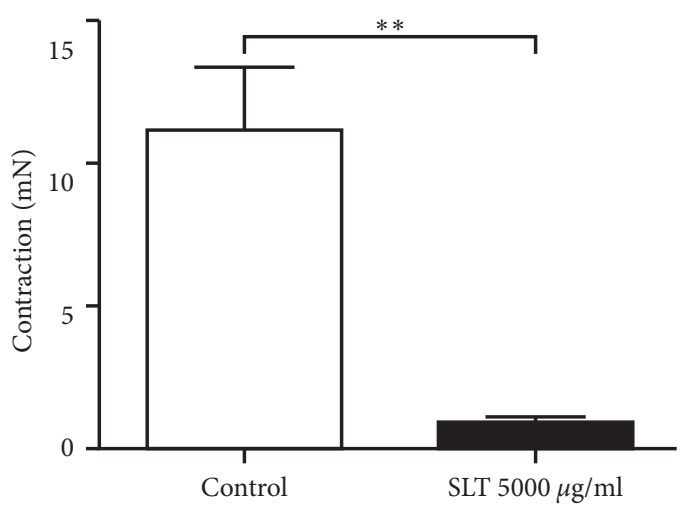

(b)

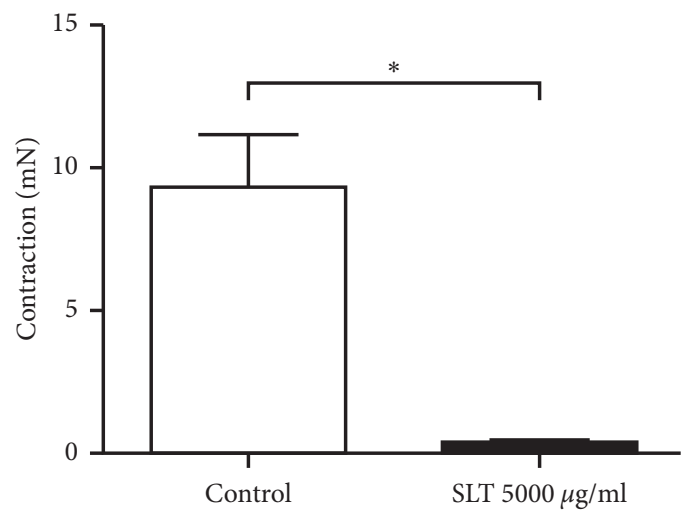

(d)

Figure 5: (a) Effect of the vehicle control (O), SLT extract $(500(\bullet), 1000(\boldsymbol{\square})$, or $5000(\mathbf{\Delta}) \mu \mathrm{g} / \mathrm{ml})$, or nifedipine $(10 \mu \mathrm{M})(\bullet)$ on phenylephrine-induced vasoconstriction in rat isolated tail arteries. Values are expressed as mean $\pm \operatorname{SEM}\left({ }^{* * *} P<0.001 \mathrm{vs}\right.$. control) $(n=5)$. (b) Isometric tension change of phenylephrine $(10 \mu \mathrm{M})$-induced vasoconstriction in rat isolated tail arteries with (closed bar) or without (open bar) the presence of the SLT extract $(5000 \mu \mathrm{g} / \mathrm{ml})$. Values are expressed as mean \pm SEM $\left({ }^{* *} P<0.01\right)(n=8)$. (c) Effect of the vehicle control $(\mathrm{O})$ or SLT extract $(500(\bullet), 1,000(\boldsymbol{\square})$, or 5,000 $(\mathbf{\Delta}) \mu \mathrm{g} / \mathrm{ml})$ on potassium chloride $(\mathrm{KCl})(80 \mathrm{mM})$-induced vasoconstriction in rat isolated tail arteries. Values are expressed as mean $\pm \operatorname{SEM}\left({ }^{*} P<0.05 ;{ }^{* *} P<0.01 ;{ }^{* * *} P<0.001\right.$ vs. control) $(n=3)$. (d) Isometric tension change of $\mathrm{KCl}(80 \mathrm{mM})$-induced vasoconstriction in rat isolated tail arteries with (closed bar) or without (open bar) the presence of the SLT extract $(5,000 \mu \mathrm{g} / \mathrm{ml})$. Values are expressed as mean \pm SEM $\left({ }^{* *} P<0.01\right)(n=3)$. 


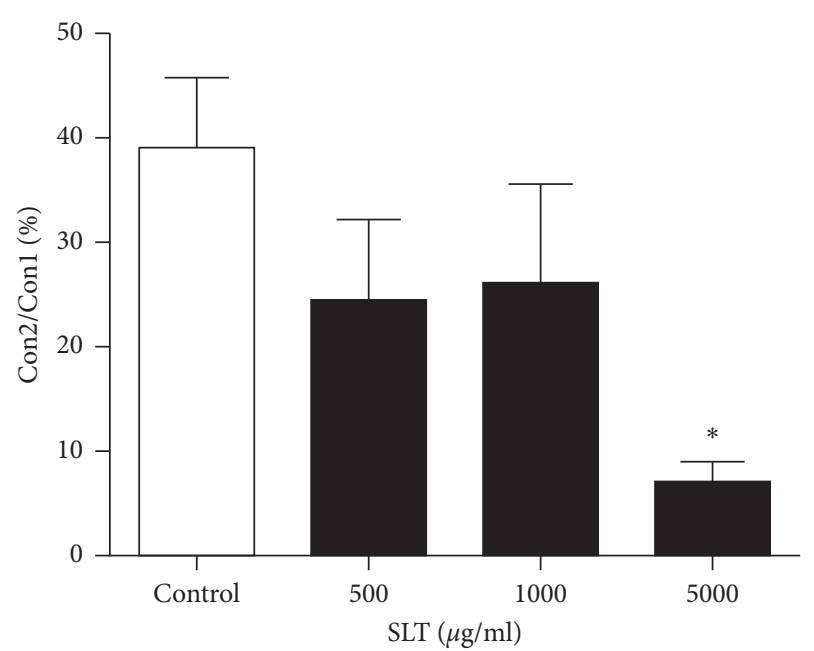

FIgURE 6: Effect of the SLT extract $(500,1,000$, and 5,000 $\mu \mathrm{g} / \mathrm{ml})$ on phenylephrine $(10 \mu \mathrm{M})$-induced transient vasoconstriction in rat isolated tail arteries. Con2/Con1 (\%) refers to the ratio of the second contraction to the first contraction. Values are expressed as mean \pm SEM $\left({ }^{*} P<0.05\right.$ vs control $)(n=6)$.

endothelium-independent mechanisms including inhibition of extracellular $\mathrm{Ca}^{2+}$ influx and release of $\mathrm{Ca}^{2+}$ storage from the sarcoplasmic reticulum.

The individual herb extracts of SLT have been shown to induce vasodilatation via endothelium-mediated mechanisms [20]. For example, crocetin, a carotenoid derived from saffron, has been shown to exert vasomodulatory effects via an endothelium-dependent mechanism [21]. Similarly, previous studies have demonstrated that ginseng produces its vasodilatory effect through eNOS activation in the endothelium $[22,23]$. In contrast, our results revealed that inhibition of the endothelium by L-NAME failed to suppress the SLT-induced vasodilatation, indicating that the SLTinduced relaxation is largely mediated by an endotheliumindependent mechanism. This relaxation was most likely caused by the complex constituents within the SLT extract which acted directly on the vascular smooth muscle cell (VSMC). For instance, the Ginkgo biloba extract (GBE) has been shown to induce vasodilation via multiple mechanisms, including inhibition of calcium influx through the L-type calcium channel and the activation of $\mathrm{NO}$ release in the rat isolated aorta [24]. Indeed, it has been shown that, while all the Ginkgo extract constituents, including bilobalide, ginkgolide $\mathrm{A}$, ginkgolide $\mathrm{B}$, ginkgolide $\mathrm{C}$, quercetin, and rutin, have vasodilating action, each of them can contribute to GBE-induced vasorelaxation with different mechanisms and signaling pathways [25].

Since our results indicate that the SLT-induced relaxation was not mediated via the endothelium, other endothelium-independent mechanisms were considered. Vascular tone and reactivity regulation are primarily controlled by the changes of intracellular calcium within the vascular smooth muscle cell (VSMC) [26]. A number of calcium channels and mechanisms are responsible for the increase of the intracellular calcium level in VSMC [27-30], and many herbs and their isolated compounds have been shown to induce vasodilatation via inhibition of calcium influx in the VSMC [24]. For instance, the Ginkgo biloba extract has been shown to induce aortic relaxation via inhibition of calcium influx through the calcium channel [24]. Similarly, ginsenoside $\operatorname{Rg}(3)$, an active ingredient of Panax ginseng, has been shown to inhibit the L-type calcium channel in Xenopus oocytes in a dose- and voltage-dependent manner [31]. In the current study, reintroduction of calcium to phenylephrine- or KCl-stimulated isolated tail artery bathed in a calcium-free solution caused contractions in a concentration-dependent manner. These results clearly demonstrated the role of calcium influx in vasoconstriction in response to both receptor-mediated (i.e., phenylephrine) and voltage-mediated $(\mathrm{KCl})$ stimulations. SLT dose-dependently inhibited the calcium-influx-induced vessel contraction to both phenylephrine- and $\mathrm{KCl}$-stimulated tail arteries, suggesting SLT was likely to inhibit both receptorand voltage-mediated calcium influx. In line with this, the ability of SLT to suppress both phenylephrine- and $\mathrm{KCl}$ mediated responses were clearly demonstrated in the phenylephrine- and $\mathrm{KCl}$-induced vasoconstriction experiments, where SLT markedly suppressed the vasoconstriction caused by both agents. Therefore, our results suggested that SLT suppressed calcium influx by inhibition of both the voltage-operated calcium channel (VOCC) and receptoroperated calcium channel (ROCC).

Although the effects of SLT on intracellular calcium storage regulation in VSMC have not been reported previously, the individual components of SLT have been shown to suppress increase of the intracellular calcium level via inhibition of both influxes of extracellular $\mathrm{Ca}^{2+}$ and release of intracellular $\mathrm{Ca}^{2+}$ storage. In our study, SLT inhibited the $\mathrm{PE}$-induced transient contraction in the isolated tail artery, indicating that SLT suppressed the vasoconstriction via inhibition of the release of $\mathrm{Ca}^{2+}$ from the sarcoplasmic reticulum. Similar to our study, ginsenoside-Rd, a main active constituent of Panax ginseng, has been shown to suppress phenylephrine-induced contraction in rat isolated aorta via inhibition of both receptor- and store-operated calcium channels in VSMC [22]. Yang et al. [32] have shown that the antiplatelet and antithrombosis properties of saffron were mediated by a mechanism involving inhibition of calcium mobilization via reducing both intracellular calcium release and extracellular calcium influx [32].

Many herbal medicines have been shown to produce their cardiovascular protective effects via the opening of potassium channels in VSMCs, such as the ATP-sensitive potassium channel $\left(\mathrm{K}_{\mathrm{ATP}}\right)$ and calcium-activated potassium channel $\left(\mathrm{K}_{\mathrm{Ca}}\right)[33,34]$. For example, $\mathrm{Li}$ et al. [35] showed that ginsenosides (GS), an extract of Panax ginseng, induced aortic relaxation and is, at least, partly mediated by the opening of the $\mathrm{K}_{\mathrm{Ca}}$ channel in the VSMC [35]. Similarly, activation of the potassium channel has also been demonstrated in ginsenoside Rg3-mediated endothelium-dependent relaxation in rat isolated aorta [36]. The Ginkgo extract has been shown to relax smooth muscle cell via a TEAsensitive (a nonselective potassium channel blocker) mechanism [37] and opening of the $\mathrm{K}_{\mathrm{Ca}}$ channel [38], while other studies suggested that Ginkgo induces vasodilatation 
via inhibition of calcium influx [24, 39] and activation of eNOS [40]. In this study, preincubation of TEA (a nonselective potassium channel blocker), glibenclamide (an ATPsensitive potassium channel blocker), and clotrimazole (a calcium-activated potassium channel blocker) did not have any effects on the SLT-induced relaxation in the rat isolated tail artery, suggesting that the relaxation was independent of potassium channels opening. Although the effects of the individual components of SLT on the potassium channel have been reported previously [36], it is not uncommon that Chinese herbal medicine can induce relaxation independent of potassium channel activation. For example, the Danshen aqueous extract and its active component, Salvianolic acid B, have been shown to produce their vasorelaxant effect independent from potassium channel opening [41].

There are three limitations in the current study. First of all, although numerous studies have demonstrated that the reactivity profile of the rat tail artery resembles the human peripheral arteries [42-44] and have been used as an ex vivo model of resistance artery which can be extrapolated to human peripheral vascular disease [45], quantitative measurement of cerebrovascular reactivity should be employed in the future study. In addition, while our current work showed that acute SLT administration did not induce endothelium-dependent vasodilatation in the vessels isolated from a healthy animal, previous work from our group has demonstrated that SLT protected the cultured human endothelial cell from oxidative injury [12], indicating that the endothelium modulatory effects of SLT can only be observed under diseased conditions. Hence, endothelial effects of SLT in disease models should be studied in the future. Finally, while the active components of SLT have been shown to be absorbed into systematic circulation [46], any effects mediated by the metabolites of SLT may be overlooked by the ex vivo model used in the current study. A detailed metabolomics study is required to identify and explore the possible contribution of metabolites of SLT on vasculature.

\section{Conclusions}

In conclusion, we demonstrated that acute administration of the SLT extract induced vasodilatation in rat isolated tail artery. SLT-induced vasodilatation appeared to be principally mediated via endothelium-independent mechanisms, including blockage of extracellular $\mathrm{Ca}^{2+}$ influx and inhibition of release of $\mathrm{Ca}^{2+}$ from the sarcoplasmic reticulum. Results from this work provide direct evidence to explain the beneficial effects of SLT observed in clinical trials.

\section{Abbreviations}

$\begin{array}{ll}\text { SLT: } & \text { Sailuotong } \\ \text { VaD: } & \text { Vascular dementia } \\ \text { WKY: } & \text { Wistar Kyoto } \\ \mathrm{Ca}^{2+}: & \text { Calcium ion } \\ \mathrm{K}^{+}: & \text {Potassium ion } \\ \mathrm{KCl}: & \text { Potassium chloride } \\ \mathrm{CaCl}_{2}: & \text { Calcium chloride } \\ \mathrm{SOD}: & \text { Superoxide dismutase }\end{array}$

$\begin{array}{ll}\text { CVD: } & \text { Cardiovascular diseases } \\ \text { Ach: } & \text { Acetylcholine } \\ \text { TEA: } & \text { Tetraethylammonium } \\ \text { EGTA: } & \text { Ethylene glycol-bis( } \beta \text {-aminoethyl ether)- } N \text {, } \\ & N, N^{\prime}, N^{\prime} \text {-tetraacetic acid } \\ \text { PE: } & \text { Phenylephrine } \\ \text { L- } & N \text {-nitroarginine methyl ester } \\ \text { NAME: } & \\ \mathrm{K}_{\text {ATP }} & \text { ATP-sensitive potassium channel } \\ \mathrm{K}_{\mathrm{Ca}}: & \text { Calcium-dependent potassium channel } \\ \text { SR: } & \text { Sarcoplasmic reticulum } \\ \text { VSMC: } & \text { Vascular smooth muscle cell } \\ \text { VOCC: } & \text { Voltage-operate calcium channel } \\ \text { ROCC: } & \text { Receptor-operate calcium channel. }\end{array}$

\section{Data Availability}

The data that support the findings of this study are available from the corresponding author (SWS) upon reasonable request.

\section{Ethical Approval}

All animal protocols conformed to the Guide for the Care and Use of Laboratory Animals by the Australian Code of Practice for the Care and Use of Animals for Scientific Purpose. Institutional ethics approval (Approval number: A12041) was obtained from Western Sydney University prior to commencement of the study.

\section{Conflicts of Interest}

As a medical research institute, NICM Health Research Institute receives research grants and donations from foundations, universities, government agencies, individuals, and industry. Sponsors and donors also provide untied funding for work to advance the vision and mission of the institute. The authors declare no conflicts of interest.

\section{Authors' Contributions}

Yeon S. Y. and Seto S. W. contributed equally to this work. SWS conceived the study. SWS and CGHH designed the study. SY and NW conducted the experiment. SY and ML performed data analysis. SWS and SY drafted this manuscript. DC, CGHH, HK, NW, and LJ revised the manuscript. All authors read and approved the final manuscript.

\section{Acknowledgments}

SY was supported by an International Postgraduate Research Scholarship, Western Sydney University, Australia. SWS was supported in part by a Cardiac Health Institute Research Fellowship. This work was partially supported by a grant (Ref no. CC-CSTH-2017-128(E)) from the College of Professional and Continuing Education, the Hong Kong Polytechnic University. 


\section{References}

[1] P. B. Gorelick, A. Scuteri, S. E. Black et al., "Vascular contributions to cognitive impairment and dementia," Stroke, vol. 42, no. 9, pp. 2672-2713, 2011.

[2] C. Y. Santos, P. J. Snyder, W. C. Wu, M. Zhang, A. Echeverria, and J. Alber, "Pathophysiologic relationship between alzheimer's disease, cerebrovascular disease, and cardiovascular risk: a review and synthesis," Alzheimer's \& Dementia: Diagnosis, Assessment \& Disease Monitoring, vol. 7, no. 1, pp. 69-87, 2017.

[3] S. W. Seto, S. M. Krishna, H. Yu, D. Liu, S. Khosla, and J. Golledge, "Impaired acetylcholine-induced endotheliumdependent aortic relaxation by caveolin-1 in angiotensin IIinfused apolipoprotein-E (ApoE-/-) knockout mice," PLoS One, vol. 8, no. 3, Article ID e58481, 2013.

[4] T. Y. Lam, S. W. Seto, Y. M. Lau et al., "Impairment of the vascular relaxation and differential expression of caveolin-1 of the aorta of diabetic $+\mathrm{db} /+\mathrm{db}$ mice," European Journal of Pharmacology, vol. 546, no. 1-3, pp. 134-141, 2006.

[5] S. W. Seto, S. Bexis, P. Aiden McCormick, and J. R. Docherty, "Actions of thalidomide in producing vascular relaxations," European Journal of Pharmacology, vol. 644, no. 1-3, pp. 113-119, 2010.

[6] B. Sharma and N. Singh, "Defensive effect of natrium diethyldithiocarbamate trihydrate (NDDCT) and lisinopril in DOCA-salt hypertension-induced vascular dementia in rats," Psychopharmacology, vol. 223, no. 3, pp. 307-317, 2012.

[7] M. R. Meyer, N. C. Fredette, M. Barton, and E. R. Prossnitz, "Prostanoid-mediated contractions of the carotid artery become Nox2-independent with aging," Age, vol. 37, no. 4, p. 79, 2015.

[8] D. Chang, J. Liu, K. Bilinski et al., "Herbal medicine for the treatment of vascular dementia: an overview of scientific evidence," Evidence-Based Complementary and Alternative Medicine, vol. 2016, Article ID 7293626, 15 pages, 2016.

[9] X. Zhou, G. Cui, and H. H. Ling Tseng, "Vascular contributions to cognitive impairment and treatments with traditional Chinese medicine," Evidence-Based Complementary and Alternative Medicine, vol. 2016, Article ID 9627258, 12 pages, 2016.

[10] J. Liu, "Development of an evidence-based Chinese herbal medicine for the management of vascular dementia," Western Sydney University, Penrith, Australia, 2008.

[11] J. Jia, C. Wei, S. Chen et al., "Efficacy and safety of the compound Chinese medicine SaiLuoTong in vascular dementia: a randomized clinical trial," Alzheimer's \& Dementia: Translational Research \& Clinical Interventions, vol. 4, no. 1, pp. 108-117, 2018.

[12] S. Seto, D. Chang, W. Ko et al., "Sailuotong prevents hydrogen peroxide $\left(\mathrm{H}_{2} \mathrm{O}_{2}\right)$-induced injury in EA.hy926 cells," International Journal of Molecular Sciences, vol. 18, no. 1, p. 95, 2017.

[13] J. Liu, D. Chan, J. Liu, and A. Bensoussan, “A randomised placebo-controlled clinical trial of a Chinese herbal medicine for the treatment of vascular dementia," in Proceedings of the 2nd International Congress for Complementary Medicine Research, Munich, Germany, 2007.

[14] G. Z. Steiner, "The effect of Sailuotong (SLT) on neurocognitive and cardiovascular function in healthy adults: a randomised, double-blind, placebo controlled crossover pilot trial," BMC Complementary and Alternative Medicine, vol. 16, no. 1, p. 15, 2015.
[15] L. Xu and J. X. Liu, "Effect of weinaokang (SLT) on dysmensia mice model," Journal of Pharmacological and Clinical Chinese Herbal Medicine, vol. 23, 2007.

[16] N. Health, M. R. Council, and A. R. Council, Australian Code for the Care and Use of Animals for Scientific Purposes, National Health and Medical Research Council, London, UK, 2013.

[17] D. M. Andrade, L. L. Borges, I. M. Torres, E. C. Conceição, and M. L. Rocha, "Jabuticaba-induced endothelium-independent vasodilating effect on isolated arteries," Arquivos Brasileiros de Cardiologia, vol. 107, no. 3, pp. 223-229, 2016.

[18] S. Seto, G. Y. Yang, H. Kiat, A. Bensoussan, Y. W. Kwan, and D. Chang, "Diabetes mellitus, cognitive impairment, and traditional Chinese medicine," International Journal of Endocrinology, vol. 2015, Article ID 810439, 14 pages, 2015.

[19] A. Yasir, T. Hardigan, and A. Ergul, "Diabetes-mediated middle cerebral artery remodeling is restored by linagliptin: interaction with the vascular smooth muscle cell endothelin system," Life Sciences, vol. 159, pp. 76-82, 2016.

[20] Y. Kubota, N. Tanaka, S. Kagota et al., "Effects of Ginkgo biloba extract on blood pressure and vascular endothelial response by acetylcholine in spontaneously hypertensive rats," Journal of Pharmacy and Pharmacology, vol. 58, no. 2, pp. 243-249, 2006.

[21] A. Mancini, J. Serrano-Díaz, E. Nava et al., "Crocetin, a carotenoid derived from saffron (Crocus sativus L.), improves acetylcholine-induced vascular relaxation in hypertension," Journal of Vascular Research, vol. 51, no. 5, pp. 393-404, 2014.

[22] Y.-Y. Guan, J.-G. Zhou, Z. Zhang et al., "Ginsenoside-Rd from panax notoginseng blocks $\mathrm{Ca}^{2+}$ influx through receptor-and store-operated $\mathrm{Ca}^{2+}$ channels in vascular smooth muscle cells," European Journal of Pharmacology, vol. 548, no. 1-3, pp. 129-136, 2006.

[23] N. D. Kim, E. M. Kim, K. W. Kang, M. K. Cho, S. Y. Choi, and S. G. Kim, "Ginsenoside Rg3 inhibits phenylephrine-induced vascular contraction through induction of nitric oxide synthase," British Journal of Pharmacology, vol. 140, no. 4, pp. 661-670, 2003.

[24] S. Nishida and H. Satoh, "Mechanisms for the vasodilations induced by Ginkgo biloba extract and its main constituent, bilobalide, in rat aorta," Life Sciences, vol. 72, no. 23, pp. 2659-2667, 2003.

[25] F. Cheung, Y. L. Siow, and O. Karmin, "Inhibition by ginkgolides and bilobalide of the production of nitric oxide in macrophages (THP-1) but not in endothelial cells (HUVEC) 1," Biochemical Pharmacology, vol. 61, no. 4, pp. 503-510, 2001.

[26] T. Akata, "Cellular and molecular mechanisms regulating vascular tone. Part 1: basic mechanisms controlling cytosolic $\mathrm{Ca}^{2+}$ concentration and the $\mathrm{Ca}^{2+}$-dependent regulation of vascular tone," Journal of Anesthesia, vol. 21, no. 2, pp. 220-231, 2007.

[27] A. P. Somlyo and A. V. Somlyo, "Signal transduction and regulation in smooth muscle," Nature, vol. 372, no. 6503, p. 231, 1994.

[28] A. V. Stanton, "Vascular network changes in the retina with age and hypertension," Journal of Hypertension, vol. 13, no. 2, pp. 1724-1728, 1995.

[29] S. W. Seto, H. Y. Lam, W. S. Lau et al., "Role of monoamine oxidases in the exaggerated 5-hydroxytryptamine-induced tension development of human isolated preeclamptic umbilical artery," European Journal of Pharmacology, vol. 605, no. 1, pp. 129-137, 2009. 
[30] H. Karaki and G. B. Weiss, "Calcium release in smooth muscle," Life Sciences, vol. 42, no. 2, pp. 111-122, 1988.

[31] J.-H. Kim, J.-H. Lee, S. M. Jeong et al., "Stereospecific effects of ginsenoside Rg3 epimers on swine coronary artery contractions," Biol Pharm Bull, vol. 29, no. 2, pp. 365-370, 2006.

[32] L. Yang, Z. Qian, Y. Yang et al., "Involvement of $\mathrm{Ca}^{2+}$ in the inhibition by crocetin of platelet activity and thrombosis formation," Journal of Agricultural and Food Chemistry, vol. 56, no. 20, pp. 9429-9433, 2008.

[33] M. G. Campos, M. Oropeza, C. Torres-Sosa, M. JiménezEstrada, and R. Reyes-Chilpa, "Sesquiterpenoids from antidiabetic Psacalium decompositum block ATP sensitive potassium channels," Journal of Ethnopharmacology, vol. 123, no. 3, pp. 489-493, 2009.

[34] H.-X. Li, S.-Y. Han, X. Ma et al., “The saponin of red ginseng protects the cardiac myocytes against ischemic injury in vitro and in vivo," Phytomedicine, vol. 19, no. 6, pp. 477-483, 2012.

[35] Z. Li, X. Chen, Y. Niwa, S. Sakamoto, and Y. Nakaya, "Involvement of $\mathrm{Ca}^{2+}$-activated $\mathrm{K}^{+}$channels in ginsenosidesinduced aortic relaxation in rats," Journal of Cardiovascular Pharmacology, vol. 37, no. 1, pp. 41-47, 2001.

[36] N. D. Kim, S. Y. Kang, J. H. Park, and V. B. Schini-Kerth, "Ginsenoside $\mathrm{Rg}_{3}$ mediates endothelium-dependent relaxation in response to ginsenosides in rat aorta: role of $\mathrm{K}^{+}$ channels," European Journal of Pharmacology, vol. 367, no. 1, pp. 41-49, 1999.

[37] J. J. Kim, D. H. Han, S. H. Lim et al., "Effects of Ginkgo biloba extracts with mirodenafil on the relaxation of corpus cavernosal smooth muscle and the potassium channel activity of corporal smooth muscle cells," Asian Journal of Andrology, vol. 13, no. 5, p. 742, 2011.

[38] M. Mesri, M. Morales-Ruiz, and E. J. Ackermann, "Suppression of vascular endothelial growth factor-mediated endothelial cell protection by survivin targeting," The American Journal of Pathology, vol. 158, no. 5, pp. 1757-1765, 2001.

[39] Y. Wang, J. Cao, and S. Zeng, "Involvement of P-glycoprotein in regulating cellular levels of Ginkgo flavonols: quercetin, kaempferol, and isorhamnetin," Journal of Pharmacy and Pharmacology, vol. 57, no. 6, pp. 751-758, 2005.

[40] A. Koltermann, A. Hartkorn, E. Koch, R. Fürst, A. M. Vollmar, and S. Zahler, "Ginkgo biloba extract EGb ${ }^{\circledR}$ 761 increases endothelial nitric oxide production in vitro and in vivo," Cellular and Molecular Life Sciences, vol. 64, no. 13, pp. 1715-1722, 2007.

[41] F. F. Y. Lam, J. H. Keung Yeung, K. M. Chan, and P. M. Yu Or, "Relaxant effects of danshen aqueous extract and its constituent danshensu on rat coronary artery are mediated by inhibition of calcium channels," Vascular Pharmacology, vol. 46, no. 4, pp. 271-277, 2007.

[42] A. K. Fouda and J. Atkinson, "Sensitivity to noradrenaline and electrical stimulation decreases with age in the rat tail artery," Naunyn Schmiedebergs Arch Pharmacol, vol. 334, no. 1, pp. 37-39, 1986.

[43] F. T. Spradley, J. J. White, W. D. Paulson, D. M. Pollock, and J. S. Pollock, "Differential regulation of nitric oxide synthase function in aorta and tail artery from 5/6 nephrectomized rats," Physiological Reports, vol. 1, no. 6, Article ID e00145, 2013.

[44] L. E. Thompson, G. J. Rinaldi, and D. F. Bohr, "Decreased activity of the sodium-calcium exchanger in tail artery of stroke-prone spontaneously hypertensive rats," Blood Vessels, vol. 27, no. 2-5, pp. 197-201, 1990.

[45] K. Wakitani, T. Takakuwa, M. Sugioka, B. Fujitani, and H. Aishita, "Inhibitory effect of OP-41483.alpha-CD, a prostacyclin analog, on peripheral vascular lesion models in rats," The Japanese Journal of Pharmacology, vol. 59, no. 1, pp. 57-63, 1992.

[46] Y. Zhang, "Pharmacokinetics and brain distribution of ginsenosides after administration of sailuotong," Zhongguo Zhong Yao Za Zhi, vol. 39, no. 2, pp. 316-321, 2014. 